\title{
Description of Micropygomyia (Silvamyia) echinatopharynx sp. nov. (Diptera: Psychodidae) a New Species of Phlebotomine Sand Fly from the State of Tocantins, Brazil
}

\author{
José Dilermando Andrade Filho/ ${ }^{+}$, Eunice A Bianchi Galati*, Welton Aires de Andrade**, \\ Alda Lima Falcão
}

Laboratório de Leishmanioses, Centro de Pesquisas René Rachou-Fiocruz, Av. Augusto de Lima 1715, 30190-002 Belo Horizonte, MG, Brasil *Departamento de Epidemiologia, Faculdade de Saúde Pública, Universidade de São Paulo, São Paulo, SP, Brasil

**Distrito Sanitário de Porto Nacional, Fundação Nacional de Saúde, Porto Nacional, TO, Brasil

During a study of the phlebotomines of the Brazilian state of Tocantins, a new species was discovered in Porto Nacional county, here described as Micropygomyia (Silvamyia) echinatopharynx sp. nov. This is only the second species of the subgenus Micropygomyia (Silvamyia) to be described.

Key words: Micropygomyia (Silvamyia) echinatopharynx sp. nov.- Phlebotominae - sand fly - Diptera - Psychodidae

The genus Micropygomyia Barretto, 1962 has as its main characteristics the palpal formula: 1.2.3.4.5 or 1.(2.4).3.5, or 1.2.4.3.5, the absence of the papilla on the third flagellomere of the setae on the anterior katepisternum margin and of the postalar bristle, and labium with united sutures. This genus is divided into four subgenera, one of them, Silvamyia Galati, 1995 being composed of only one species, Micropygomyia (Silvamyia) acanthopharynx (Martins Falcão \& Silva, 1962), which is characterized by well developed teeth in the pharynx, an AIII longer than the head (male) or about 0.9 times its length (female) and a gonostyle with only one apical spine.

Thirty-five species have so far been reported in Tocantins, the new species having been captured in Porto Nacional county in orchards and around houses (Andrade Filho et al. 2001). We present the following description of the new species, named Micropygomyia (Silvamyia) echinatopharynx sp. nov., this name referring to the large number of spines in the pharynx.

The measurements are given in micrometers, with the mean and standard deviation values for the paratypes in parentheses. Several characters have been submitted to variance analysis (ANOVA) at the 5\% significance level. For some other characters the Mann-Withney Test was used when it was impossible to apply ANOVA, that is to say, when the samples did not meet the necessary presupposition for the latter to be used; the values did not present normal distribution, constant variation or independence. The classification follows that proposed by Galati (1995) and the terminology of the characters is in accordance with Galati (2003).

\footnotetext{
Financial supporte: Fundação Nacional de Saúde, Fiocruz +Corresponding author. Fax: +55-31-3295.3566. E-mail: jandrade@cpqrr.fiocruz.br

Received 23 June 2004

Accepted 3 September 2004
}

\section{DESCRIPTION \\ Micropygomyia (Silvamyia) echinatopharynx sp. nv.} (Figs 1-23).

Holotype female: total length $2650(2676 \pm 60.0, \mathrm{n}=12)$. Coloration pale chestnut, the pleurae being paler than the notum.

Head (Table I): length about 1.13 times width $(1.05 \pm 0.07$; $\mathrm{n}=20)$. Ratio clypeus/head 0.33:1 $(0.34 \pm 0.01 ; \mathrm{n}=20)$; eyes/head 0.53: $1(0.51 \pm 0.02 ; n=20)$; labrum-epipharynx/ head $0.54(0.54 \pm 0.02 ; n=20)$; AIII very long, labrumepipharynx/AIII $0.65(0.64 \pm 0.03 ; \mathrm{n}=16)$; AIII/head 0.87:1 (0.91: $1 \pm 0.04 ; n=16)$. Antennal formula AIII-AXV 2; AXVI 0. AIII, AIV and AV, with internal ascoid implanted at a more basal level than the external one. Ascoids reaching beyond the middle of the segment, without posterior prolongation. Final segments lost in the holotype, papillae being present in the paratypes on segments AXIV, AXV, and AXVI. Palpal formula 1.2.4.3.5. (1.2.4.3.5; $\mathrm{n}=$ 20), palpomere 3 being slightly longer than 4 . Newstead's spines implanted in a group on the basal half of the third palpomere. Cibarial arch complete and pigment patch pale but easy to see. Four horizontal teeth, little developed, and in the form of a palisade. Pharynx with numerous spines and teeth throughout its apical region. Lacinia of the maxillae with the external teeth positioned in a longitudinal row.

Cervix: ventro-cervical sensillae present.

Thorax (Table III): coloration pale chestnut on the notum, katepisternum and coxae, other areas of the pleurae being pale. Proepimeral and upper anepisternal bristles present. Setae on the anterior katepisternum margin absent. Legs without special characteristics. Wings as in the figure. Abdomen (Table V): 1470 long $(1508 \pm 53$; $\mathrm{n}=11)$. Spermathecae with approximately 10 annulations, poorly defined, 30 long $(27 \pm 2 ; n=19)$ by 10 wide $(11 \pm 1 ; n=19)$. Ducts visible only in the holotype, the individual ducts being about $4 \mathrm{x}$ longer than the common duct, measuring 176 and 44 long respectively. Cerci simple, 158 long (151 \pm 


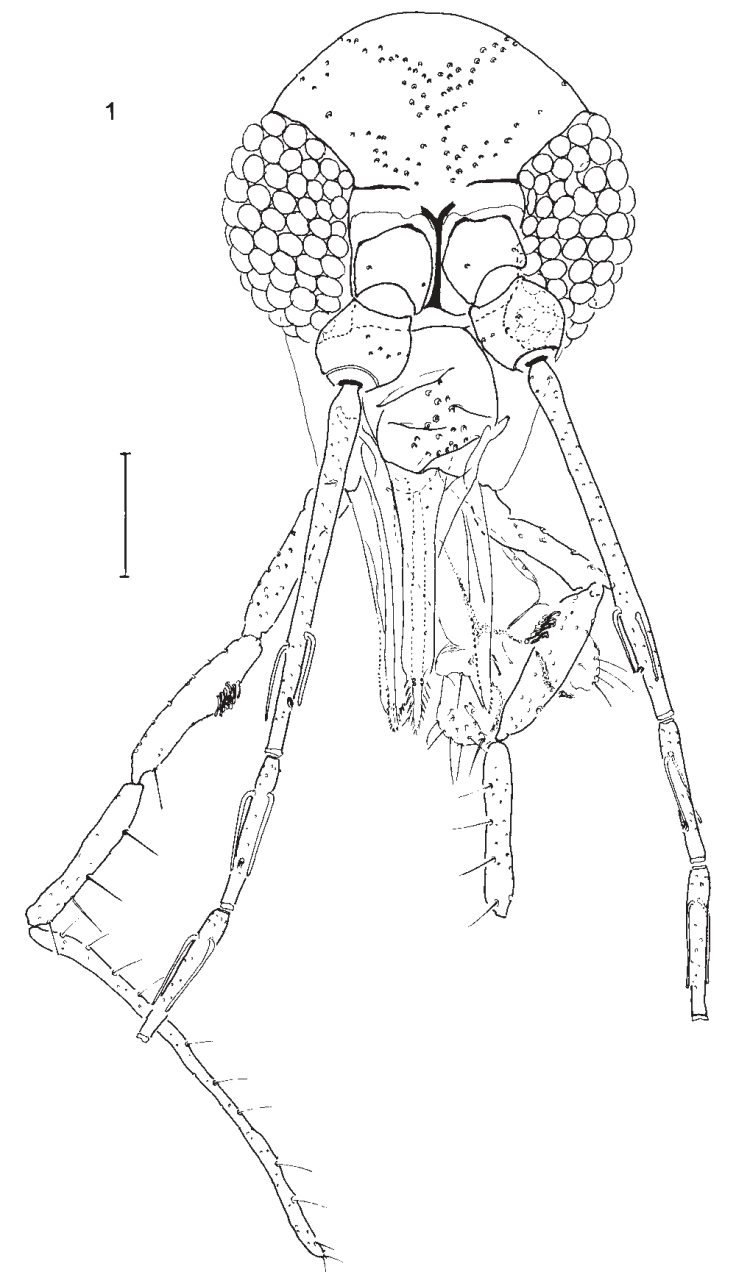

Fig. 1: Micropygomyia (Silvamyia) echinatopharynx sp. nov. Holotype o - head. $\mathrm{Bar}=100 \mu \mathrm{m}$.
$10 ; n=17)$.

Allotype male: total length $2415(2444 \pm 137.7 ; \mathrm{n}=8)$. Coloration as in the female.

Head (Table II): length 1.22 times width $(1.18 \pm 0.06 ; \mathrm{n}=$ 10). Ratio clypeus/head $0.33: 1(0.35 \pm 0.02 ; \mathrm{n}=10)$; eyes/ head 0.49:1 $(0.50 \pm 0.02 ; \mathrm{n}=9)$; labrum-epipharynx/head $0.52(0.51 \pm 0.02 ; \mathrm{n}=9)$; AIII very long, ratio labrum-epipharynx/AIII $0.60(0.55 \pm 0.05 ; n=5) \mathrm{AIII} /$ head 0.87:1 $(1.00 \pm$ $0.06 ; n=5)$. Antennal formula, distribution of the sensillae and position of the ascoids as in the female, distal prolongation of the ascoids being slightly shorter than in the females, reaching the middle of the flagellomere. Palpal formula 1.2.4.3.5 (1.2.4.3.5; $\mathrm{n}=10)$. Distribution of Newstead's spines as in the females.

Cervix: ventro-cervical sensillae present.

Thorax (Table IV): coloration similar to that of the female. Proepimeral and upper anepisternal bristles present. Setae on the anterior katepisternum margin absent. Legs without special characters. Wings as in figure.

Abdomen (Table V): length 1360 (1424 \pm 117 ; $\mathrm{n}=9$ ). Gonocoxite without tuft of setae, measuring 233 long (231 $\pm 11 ; \mathrm{n}=11)$ by 68 wide $(66 \pm 4 ; \mathrm{n}=11)$. Gonostyle 148 long $(145 \pm 7 ; n=11)$, presenting four well-developed spines, i.e., one apical, one upper external implanted on the preapical region, one lower external implanted on the apical third and one internal implanted in the middle of the structure. Paramere simple, measuring 148 (148 \pm 8 ; $n=$ 11). Lateral lobe 203 long $(212 \pm 15 ; \mathrm{n}=11)$ by 28 wide $(24$ $\pm 2 ; \mathrm{n}=11)$. Aedeagus conical. Ejaculatory pump 118 long $(117 \pm 7 ; \mathrm{n}=10)$. Genital filament measuring 540 (508 \pm 28 ; $\mathrm{n}=11)$. Ratio genital filament/ ejaculatory pump 4.58 (4.37 $\pm 0.26 ; n=10)$. Tips of genital filaments simple.

Collecting and storage of type material: holotype female, number 77361, captured on 28/08/2000, about $3 \mathrm{~km}$ from

\section{TABLE I}

Measurements $(\mu \mathrm{m})$ of the structures of the head and its appendages in females of Micropygomyia (Silvamyia) echinatopharynx sp. nov. and Micropygomyia (Silvamyia) acanthopharynx

\begin{tabular}{|c|c|c|c|c|c|c|c|c|c|}
\hline \multirow[b]{3}{*}{ Structure } & \multirow[b]{3}{*}{ Holotype } & \multirow{2}{*}{\multicolumn{3}{|c|}{$\begin{array}{c}\text { M. echinatopharynx } \\
\text { Paratypes female }\end{array}$}} & \multirow{2}{*}{\multicolumn{3}{|c|}{$\begin{array}{l}\text { M. acanthopharynx } \\
\text { Paratypes female }\end{array}$}} & \multirow{2}{*}{\multicolumn{2}{|c|}{ Anova }} \\
\hline & & & & & & & & & \\
\hline & & $X$ & $\mathrm{SD}$ & $\mathrm{N}$ & $X$ & SD & $\mathrm{N}$ & $\mathrm{F}_{\mathrm{tab}}$ & $\mathrm{F}_{\mathrm{obs}}$ \\
\hline Head length & 360 & 358 & 12 & 20 & 381 & 19 & 6 & 0,05 & $0,0047^{a}$ \\
\hline Head width & 320 & 342 & 24 & 20 & 321 & 27 & 4 & 4,28 & 2,31 \\
\hline Interocular & 128 & 130 & 5 & 20 & 122 & 14 & 4 & 4,28 & $5,37^{a}$ \\
\hline Clypeus length & 120 & 122 & 6 & 21 & 139 & 10 & 4 & 4,26 & $27,93^{a}$ \\
\hline Eye length & 190 & 183 & 9 & 20 & 190 & 8 & 4 & 4,28 & 1,81 \\
\hline Eye width & 93 & 106 & 8 & 20 & 109 & 10 & 4 & 4,28 & 0,66 \\
\hline $\mathrm{LE}$ & 193 & 195 & 6 & 21 & 216 & 19 & 4 & 4,26 & $21,09^{a}$ \\
\hline AIII & 295 & 306 & 16 & 17 & 320 & 23 & 6 & 4,32 & 2,69 \\
\hline AIV & 125 & 128 & 6 & 16 & 133 & 10 & 6 & 4,32 & 2,20 \\
\hline AV & 120 & 129 & 6 & 17 & 133 & 12 & 6 & 4,30 & 1,13 \\
\hline $\mathrm{AXV}$ & & 64 & 3 & 15 & 65 & 4 & 5 & 0,05 & 0,7270 \\
\hline AXVI & & 64 & 3 & 15 & 66 & 6 & 5 & 0,05 & 0,6005 \\
\hline P1 & 35 & 35 & 2 & 21 & 38 & 3 & 5 & 0,05 & 0,0805 \\
\hline P2 & 100 & 99 & 6 & 21 & 95 & 5 & 5 & 4,24 & 2,84 \\
\hline P3 & 150 & 145 & 9 & 20 & 149 & 11 & 6 & 0,05 & 0,9767 \\
\hline P4 & 140 & 136 & 7 & 20 & 147 & 12 & 5 & 4,26 & $7,08^{a}$ \\
\hline P5 & 353 & 339 & 21 & 19 & 346 & 36 & 5 & 4,28 & 0,26 \\
\hline
\end{tabular}

a: statistically significant; head length, AXV, AXVI; P1, P3: Mann-Whitney Test 


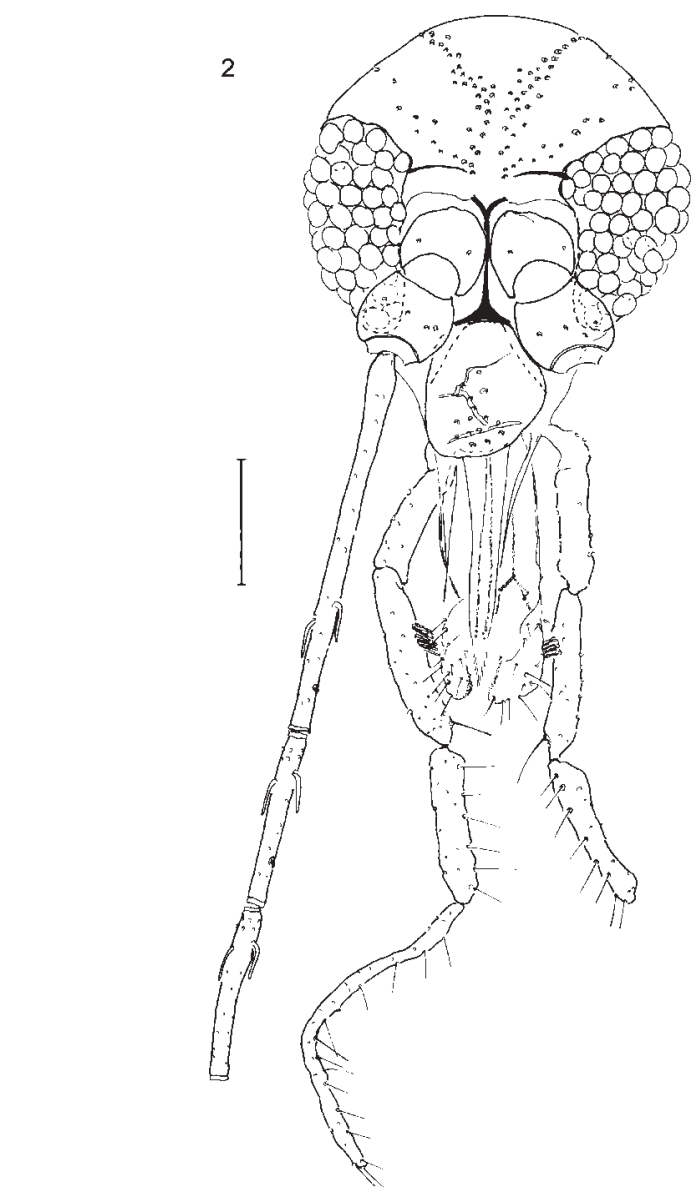

Fig. 2: Micropygomyia (Silvamyia) echinatopharynx sp. nov. Allotype $\sigma^{*}$ - head. Bar $=100 \mu \mathrm{m}$. the Tocantins river on the Fazenda Serragem, in Porto Nacional county, state of Tocantins; allotype male number 73950, 23/07/1998, in a chicken house on the Fazenda Mata Grande, Porto Nacional, Tocantins; six female paratypes, numbers 73909-73914 and four male paratypes, numbers 73905-73908, 23/07/1998, between piles of stones, close to a corral on the Fazenda Mata Grande, Porto Nacional, Tocantins; twelve female paratypes, numbers 73951-73962 and five male paratypes, numbers 73945$73949,23 / 07 / 1998$, in a chicken house on the Fazenda Mata Grande, Porto Nacional, Tocantins; two female paratypes, numbers 74092 and 74093, 27/07/1998, in an orchard on the Fazenda Mutum, Porto Nacional, Tocantins; one male paratype, number 77360, 28/08/2000, about $3 \mathrm{~km}$ from Tocantins River on the Fazenda Serragem, Porto Nacional, Tocantins; one female paratype, number 77497 and one male paratype number $77496,28 / 08 / 2000$, in a chicken house on the Fazenda Serragem, Porto Nacional, Tocantins. The capture on 17/07/1998, in the Fazenda Mutum was made with Falcão light trap and the others were made with CDC light traps.

Holotype and allotype have been deposited in the phlebotomine collection of the Centro de Pesquisas René Rachou-Fiocruz, Belo Horizonte, Minas Gerais, together with 18 female and eight male paratypes. One pair of paratypes has been deposited in the collection of the Instituto Oswaldo Cruz, Rio de Janeiro, RJ. One pair of paratypes has been deposited in the collection of the Instituto Nacional de Pesquisa da Amazônia, Manaus, Amazonas. One pair of paratypes has been deposited in the collection of the Faculdade de Saúde Pública da Universidade de São Paulo, São Paulo, SP.

TABLE II

Measurements $(\mu \mathrm{m})$ of the structures of the head and its appendages in males of Micropygomyia (Silvamyia) echinatopharynx sp. nov. and Micropygomyia (Silvamyia) acanthopharynx

\begin{tabular}{|c|c|c|c|c|c|c|c|c|c|}
\hline \multirow[b]{3}{*}{ Structure } & \multirow[b]{3}{*}{ Allotype } & \multirow{2}{*}{\multicolumn{3}{|c|}{$\begin{array}{c}\text { M. echinatopharynx } \\
\text { Paratypes female }\end{array}$}} & \multirow{2}{*}{\multicolumn{3}{|c|}{$\begin{array}{l}\text { M. acanthopharynx } \\
\text { Paratypes female }\end{array}$}} & \multirow{2}{*}{\multicolumn{2}{|c|}{ Anova }} \\
\hline & & & & & & & & & \\
\hline & & $X$ & SD & $\mathrm{N}$ & $\mathrm{X}$ & SD & $\mathrm{N}$ & $F_{\text {tab }}$ & $\mathrm{F}_{\text {obs }}$ \\
\hline Head length & 345 & 339 & 15 & 10 & 383 & 16 & 2 & 4,84 & $15,80^{a}$ \\
\hline Head width & 283 & 284 & 18 & 9 & 259 & & 1 & 5,12 & 1,96 \\
\hline Interocular & 120 & 114 & 7 & 9 & 124 & & 1 & 5,12 & 1,60 \\
\hline Clypeus length & 115 & 118 & 9 & 10 & 149 & 6 & 4 & 4,67 & $43,38^{a}$ \\
\hline Eye length & 170 & 168 & 12 & 9 & 152 & & 1 & 5,12 & 1,79 \\
\hline Eye width & 83 & 88 & 6 & 10 & 83 & & 1 & 4,96 & 0,49 \\
\hline $\mathrm{LE}$ & 180 & 183 & 12 & 10 & 205 & 12 & 4 & 4,67 & $11,38^{a}$ \\
\hline AIII & 300 & 344 & 30 & 6 & 344 & 52 & 5 & 4,96 & 3,06 \\
\hline AIV & 138 & 148 & 8 & 6 & 161 & 6 & 5 & 4,96 & $11,91^{a}$ \\
\hline $\mathrm{AV}$ & 140 & 149 & 7 & 6 & 161 & 7 & 5 & 4,96 & $10,36^{a}$ \\
\hline AXV & 58 & 64 & 4 & 3 & 66 & 8 & 5 & 5,59 & 0,82 \\
\hline AXVI & 63 & 59 & 5 & 3 & 65 & 7 & 5 & 5,59 & 1,24 \\
\hline $\mathrm{P} 1$ & 33 & 34 & 3 & 11 & 36 & 3 & 5 & 0,05 & 0,23 \\
\hline P2 & 98 & 98 & 6 & 11 & 101 & 9 & 5 & 4,54 & 0,57 \\
\hline P3 & 138 & 145 & 8 & 11 & 150 & 11 & 5 & 4,54 & 2,07 \\
\hline $\mathrm{P} 4$ & 120 & 129 & 7 & 10 & 146 & 9 & 5 & 4,60 & $17,23^{a}$ \\
\hline P5 & 263 & 275 & 18 & 10 & 298 & & 1 & 4,96 & 1,76 \\
\hline
\end{tabular}

$a$ : statistically significant; P1: Mann-Whitney Test 

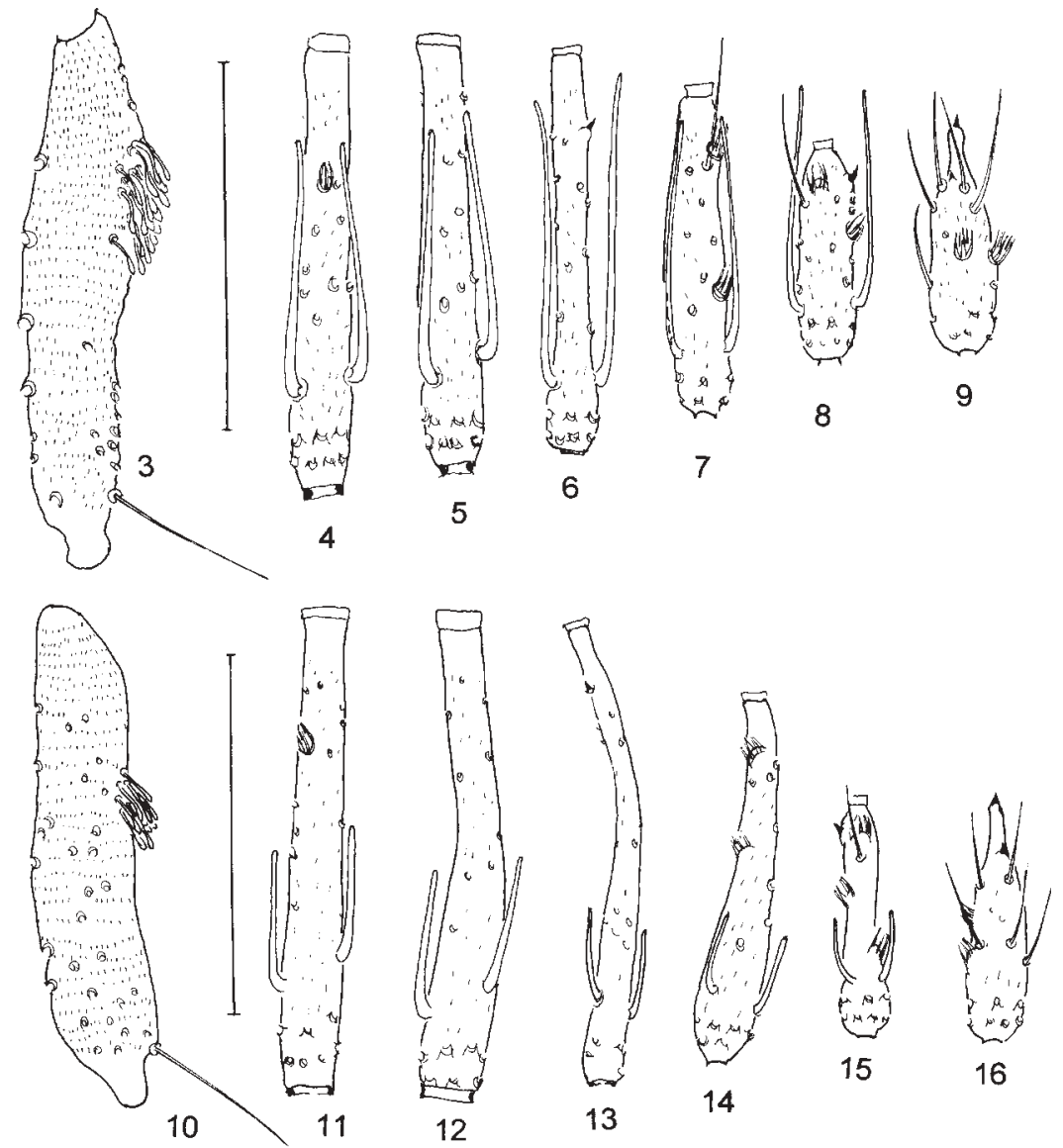

Figs 3-16: Micropygomyia (Silvamyia) echinatopharynx sp. nov. Fig. 3: holotype ㅇ - palpomere III. Fig. 4: holotype ㅇ - AIV. Fig. 5: holotype + - AV. Fig. 6: paratype + - AXIII. Fig. 7: paratype + - AXIV. Fig. 8: paratype + - AXV. Fig. 9: paratype + - AXVI. Fig. 10: allotype $\sigma^{\star}$ - palpomere III. Fig. 11: allotype $\sigma^{\star}$ - AIV. Fig. 12: allotype $\sigma^{\star}$ - AV. Fig. 13: paratype $\sigma^{\star}$ - AXIII. Fig. 14: paratype $\sigma^{\star}-$ AXIV. Fig. 15: paratype $\sigma^{*}-$ AXV. Fig 16: paratype $\sigma^{\star}-$ AXVI. Bars $=100 \mu \mathrm{m}$

TABLE III

Measurements $(\mu \mathrm{m})$ of the structures of the thorax in females of Micropygomyia (Silvamyia) echinatopharynx sp. nov. and Micropygomyia (Silvamyia) acanthopharynx

\begin{tabular}{|c|c|c|c|c|c|c|c|c|c|}
\hline \multirow[b]{3}{*}{ Structure } & \multirow[b]{3}{*}{ Holotype } & \multirow{2}{*}{\multicolumn{3}{|c|}{$\begin{array}{c}\text { M. echinatopharynx } \\
\text { Paratypes female }\end{array}$}} & \multirow{2}{*}{\multicolumn{3}{|c|}{$\begin{array}{l}\text { M. acanthopharynx } \\
\text { Paratypes female }\end{array}$}} & \multirow{2}{*}{\multicolumn{2}{|c|}{ Anova }} \\
\hline & & & & & & & & & \\
\hline & & $X$ & $\mathrm{SD}$ & $\mathrm{N}$ & $X$ & $\mathrm{SD}$ & $\mathrm{N}$ & $\mathrm{F}_{\mathrm{tab}}$ & $\mathrm{F}_{\mathrm{obs}}$ \\
\hline Wing width & 560 & 550 & 26 & 21 & 590 & 42 & 6 & 4,22 & $8,17^{a}$ \\
\hline Alpha & 400 & 411 & 40 & 21 & 442 & 46 & 5 & 0,05 & 0,0981 \\
\hline Beta & 310 & 314 & 27 & 21 & 336 & 30 & 5 & 4,24 & 2,74 \\
\hline Gamma & 230 & 233 & 20 & 21 & 239 & 35 & 5 & 4,24 & 0,29 \\
\hline Delta & 90 & 87 & 21 & 21 & 129 & 34 & 5 & 4,24 & $12,76^{a}$ \\
\hline R5 & 1240 & 1244 & 48 & 21 & 1325 & 81 & 5 & 4,24 & $9,20^{a}$ \\
\hline Anterior femur & 770 & 750 & 35 & 12 & 789 & 69 & 3 & 4,67 & 2,06 \\
\hline Median femur & & 721 & 25 & 14 & 755 & 49 & 4 & 4,49 & 3,67 \\
\hline Posterior femur & & 793 & 29 & 14 & 834 & 59 & 3 & 4,54 & 3,44 \\
\hline Anterior tibia & 1000 & 1040 & 46 & 12 & 1033 & 86 & 3 & 4,60 & 0,01 \\
\hline Mean tibiae & & 1169 & 56 & 14 & 1175 & 85 & 4 & 4,49 & 0,03 \\
\hline Posterior tibia & & 1376 & 73 & 14 & 1390 & 104 & 3 & 4,54 & 0,08 \\
\hline First anterior tarsomere & 630 & 648 & 28 & 11 & 654 & 49 & 3 & 4,67 & 0,12 \\
\hline First median tarsomere & & 671 & 30 & 14 & 682 & 59 & 4 & 4,49 & 0,27 \\
\hline First posterior tarsomere & & 742 & 41 & 14 & 774 & 86 & 3 & 4,54 & 1,06 \\
\hline Anterior tarsomeres $2+3+4+5$ & 730 & 747 & 29 & 11 & 721 & 49 & 3 & 4,67 & 1,36 \\
\hline Median tarsomeres $2+3+4+5$ & & 740 & 36 & 14 & 704 & 45 & 4 & 4,49 & 2,72 \\
\hline Posterior tarsomeres $2+3+4+5$ & & 776 & 33 & 14 & 747 & 46 & 3 & 4,54 & 1,69 \\
\hline
\end{tabular}

$a$ : statistically significant; alpha: Mann-Whitney Test 


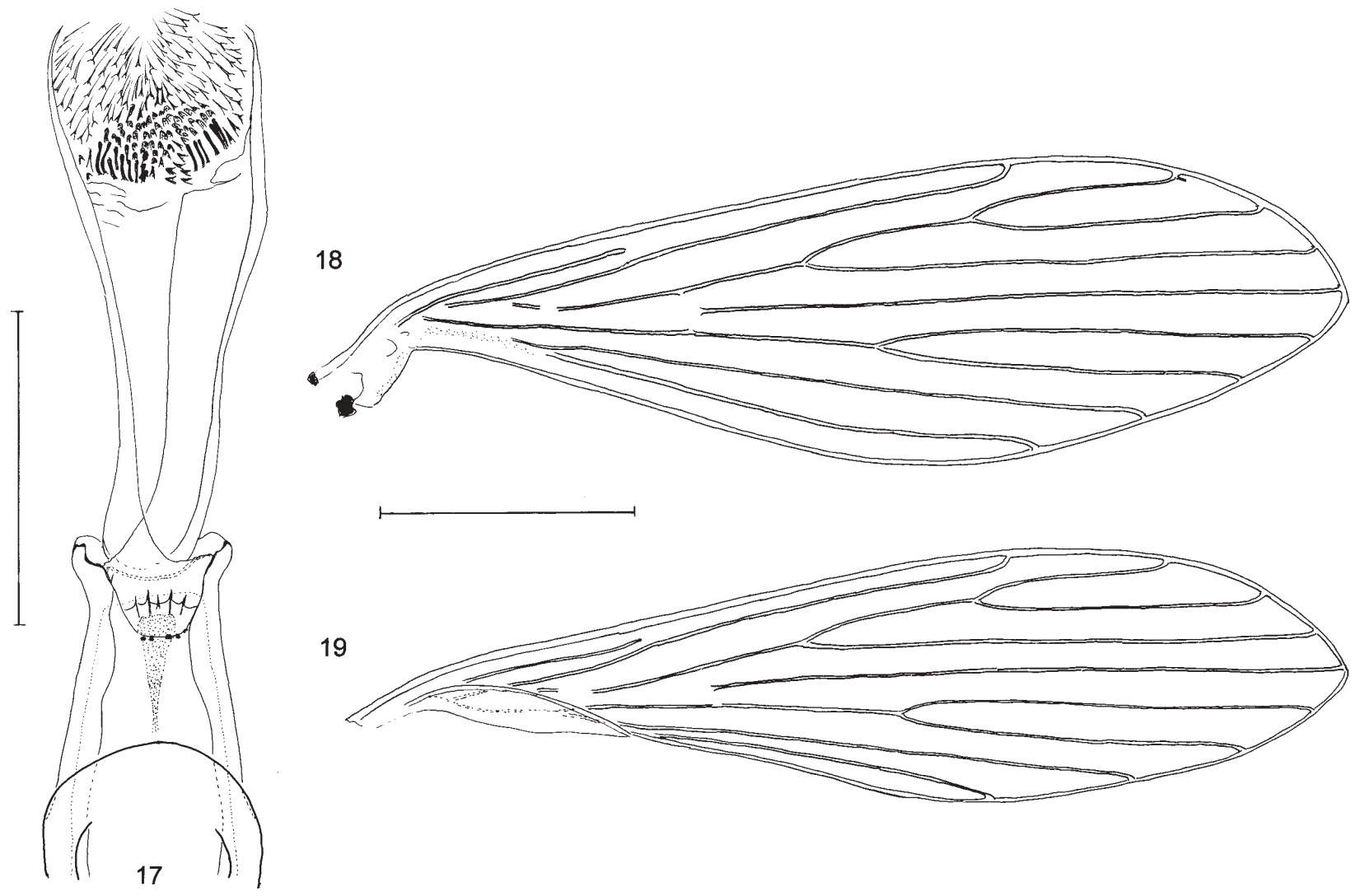

Fig. 17: Micropygomyia (Silvamyia) echinatopharynx sp. nov. Holotype + - cibarium and pharynx. Bar $=100 \mu \mathrm{m}$. Figs 18-19: Micropygomyia (Silvamyia) echinatopharynx sp. nov. Fig. 18: holotype + - wing. Fig.19: allotype $\delta^{\top}-$ wing. Bar $=500 \mu \mathrm{m}$

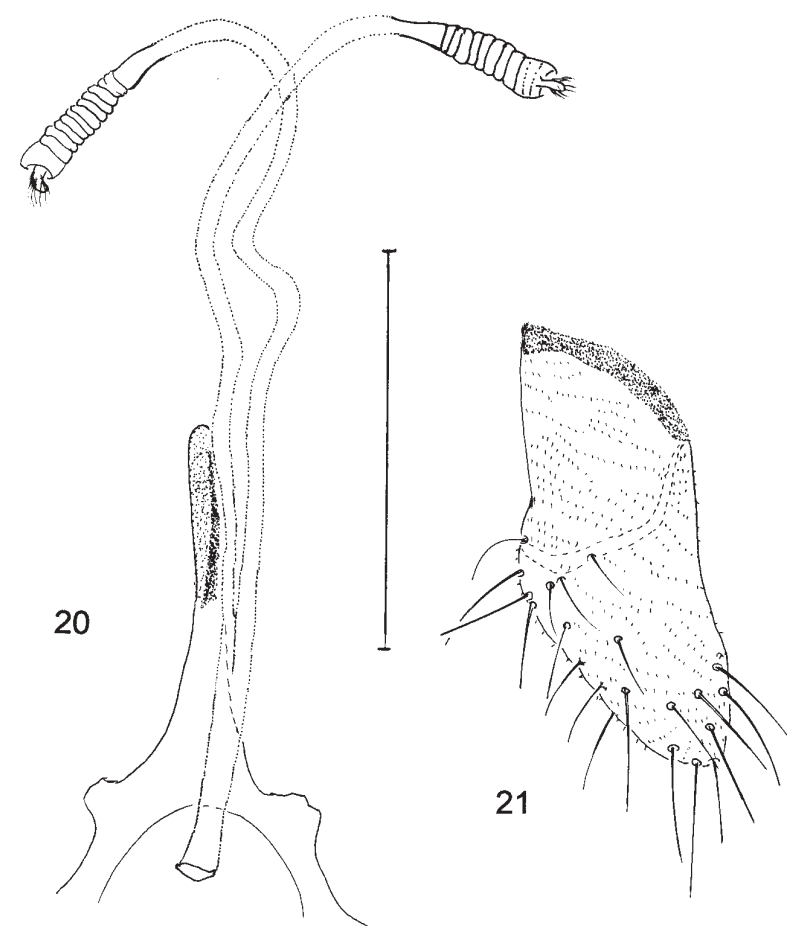

Figs 20-21: Micropygomyia (Silvamyia) echinatopharynx sp. nov. Fig. 20: holotype + - genital fork and spermathecae. Fig. 21: holotype + - cercus. Bar $=100 \mu \mathrm{m}$

\section{REMARKS}

The only species belonging at present to the subgenus Silvamyia is $M$. acanthopharynx. M. echinatophar$y n x \mathrm{n}$. sp. and M. acanthopharynx may be distinguished by several female and male characteristics (Tables I-V), among which the length of the clypeus, labrum-epipharynx and width of the wing are noteworthy, all been greater in the latter species. In the male terminalia, only the genital filaments of the two species present no difference at the $5 \%$ level, the ratio between the length of this structure and that of the ejaculatory pump being always greater than 4.12 in M. echinatopharynx and less than 3.97 in $M$. acanthopharynx. The position of the spines on the gonostyle permits a clear distinction to be made between the two species: in M. acanthopharynx the lower external spine and the internal one are located at the same level, while in new species they are at different levels, the lower external spine being in the apical third and the internal one in the middle of the gonostyle. The female of the new species can be distinguished clearly from that of M. acanthopharynx by the cibarial teeth, less developed in the new species.

The two species were captured in the same ecotopes, on the savanna land of the central region of Brazil (Martins et al. 1962, Andrade Filho et al. 2001), which shows that M. echinatopharynx is really a different species from $M$. acanthopharynx and that they are not intraspecific varieties. 


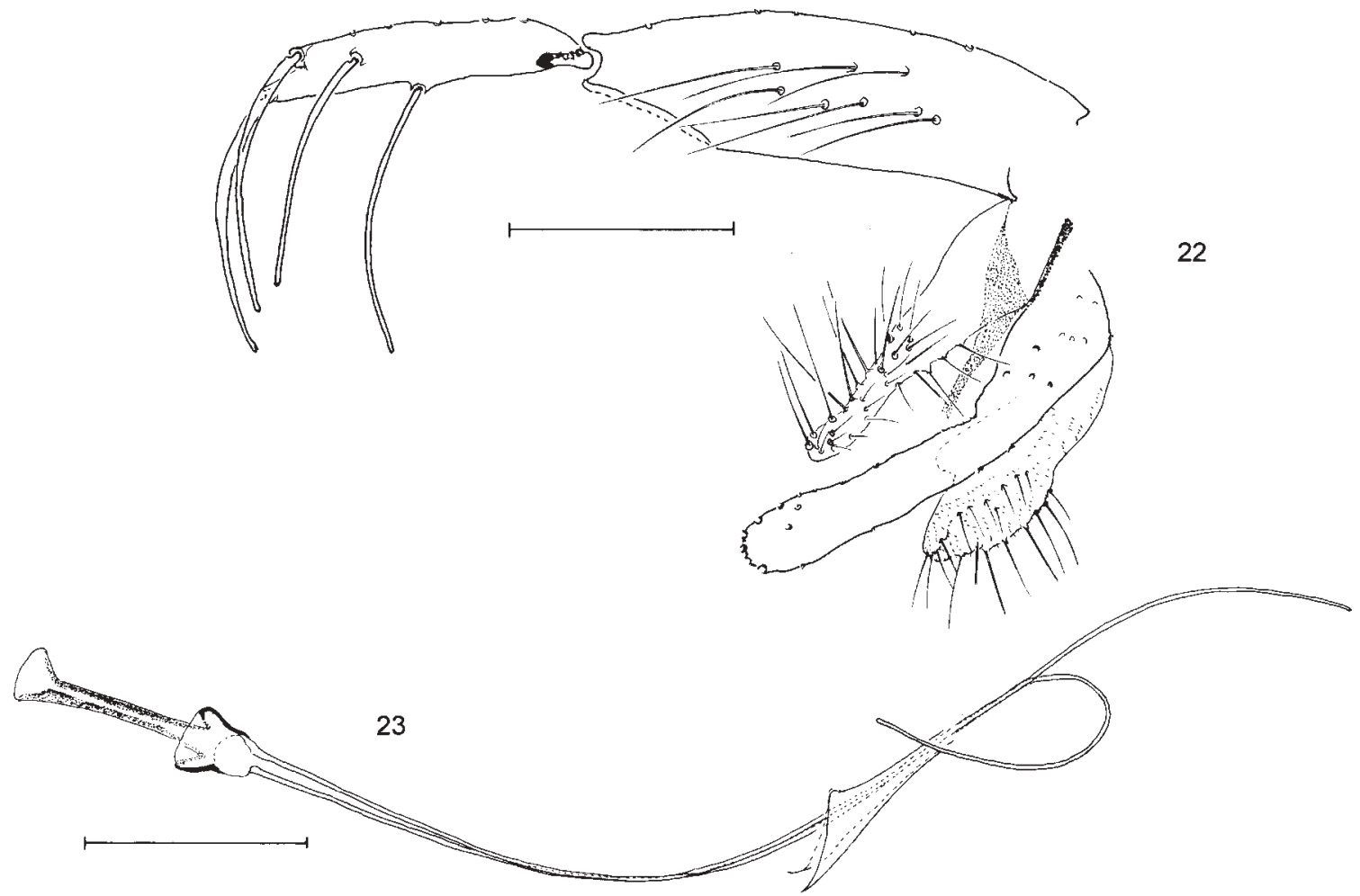

Figs 22-23: Micropygomyia (Silvamyia) echinatopharynx sp. nov. Fig. 22: allotype $\sigma^{\top}-$ terminalia. Fig. 23: allotype $\delta^{\star}-$ genital pump and filaments. Bars $=100 \mu \mathrm{m}$

TABLE IV

Measurements $(\mu \mathrm{m})$ of the structures of the thorax in males of Micropygomyia (Silvamyia) echinatopharynx sp. nov. and Micropygomyia (Silvamyia) acanthopharynx

\begin{tabular}{|c|c|c|c|c|c|c|c|c|c|}
\hline \multirow[b]{3}{*}{ Structure } & \multirow[b]{3}{*}{ Aloparatype } & \multirow{2}{*}{\multicolumn{3}{|c|}{$\begin{array}{c}\text { M. echinatopharynx } \\
\text { Paratypes female }\end{array}$}} & \multirow{2}{*}{\multicolumn{3}{|c|}{$\begin{array}{l}\text { M. acanthopharynx } \\
\text { Paratypes female }\end{array}$}} & \multirow{2}{*}{\multicolumn{2}{|c|}{ Anova }} \\
\hline & & & & & & & & & \\
\hline & & $\mathrm{X}$ & $\mathrm{SD}$ & $\mathrm{N}$ & $\mathrm{X}$ & $\mathrm{SD}$ & $\mathrm{N}$ & $\mathrm{F}_{\mathrm{tab}}$ & $\mathrm{F}_{\mathrm{obs}}$ \\
\hline Wing width & & 444 & 28 & 7 & 516 & 56 & 5 & 4,96 & $8,71^{a}$ \\
\hline Alpha & & 348 & 38 & 3 & 399 & 63 & 5 & 5,59 & 2,06 \\
\hline Beta & & 303 & 5 & 4 & 320 & 38 & 5 & 5,59 & 0,82 \\
\hline Gamma & & 210 & 30 & 3 & 241 & 33 & 5 & 5,99 & 1,82 \\
\hline Delta & & 38 & 17 & 4 & 68 & 57 & 5 & 5,99 & $7,18^{a}$ \\
\hline R5 & & 1100 & 110 & 4 & 1251 & 117 & 5 & 5,59 & 3,90 \\
\hline Anterior femur & 710 & 729 & 26 & 7 & 706 & 54 & 2 & 5,32 & 0,72 \\
\hline Median femur & 690 & 678 & 30 & 6 & 781 & 64 & 3 & 5,32 & $14,44^{a}$ \\
\hline Posterior femur & 760 & 760 & 50 & 4 & 823 & 87 & 4 & 5,59 & 2,04 \\
\hline Anterior tibia & 1020 & 1071 & 62 & 7 & 1048 & 127 & 2 & 5,32 & 0,58 \\
\hline Mean tibiae & 1160 & 1184 & 82 & 5 & 1225 & 115 & 3 & 5,59 & 3,87 \\
\hline Posterior tibia & 700 & 728 & 54 & 4 & 1418 & 154 & 4 & 5,59 & 4,00 \\
\hline First anterior tarsomere & 650 & 658 & 29 & 6 & 660 & 56 & 2 & 5,59 & 0,01 \\
\hline First median tarsomere & 660 & 656 & 43 & 5 & 699 & 49 & 3 & 5,59 & 2,02 \\
\hline First posterior tarsomere & 700 & 728 & 54 & 4 & 769 & 62 & 4 & 5,59 & 1,65 \\
\hline Anterior tarsomeres $2+3+4+5$ & 720 & 728 & 33 & 6 & 688 & 64 & 2 & 5,59 & 1,77 \\
\hline Median tarsomeres $2+3+4+5$ & 720 & 718 & 29 & 5 & 706 & 58 & 3 & 5,59 & 0,21 \\
\hline Posterior tarsomeres $2+3+4+5$ & & 745 & 38 & 4 & 755 & 60 & 3 & 6,61 & 0,07 \\
\hline
\end{tabular}

a: statistically significant 
TABLE V

Measurements $(\mu \mathrm{m})$ of the structures of the abdomen in males and females of Micropygomyia (Silvamyia) echinatopharynx sp. nov. and Micropygomyia (Silvamyia) acanthopharynx

\begin{tabular}{|c|c|c|c|c|c|c|c|c|c|}
\hline \multirow[b]{3}{*}{ Structure } & \multirow[b]{3}{*}{ Holotype } & \multirow{2}{*}{\multicolumn{3}{|c|}{$\begin{array}{c}\text { M. echinatopharynx } \\
\text { Paratypes female }\end{array}$}} & \multirow{2}{*}{\multicolumn{3}{|c|}{$\begin{array}{l}\text { M. acanthopharynx } \\
\text { Paratypes female }\end{array}$}} & \multirow{2}{*}{\multicolumn{2}{|c|}{ Anova }} \\
\hline & & & & & & & & & \\
\hline & & $X$ & SD & $\mathrm{N}$ & $X$ & SD & $\mathrm{N}$ & $\mathrm{F}_{\mathrm{tab}}$ & $\mathrm{F}_{\mathrm{obs}}$ \\
\hline Spermatheca length & 30 & 27 & 2 & 19 & & & & & \\
\hline Spermatheca width & 10 & 11 & 1 & 19 & & & & & \\
\hline \multirow[t]{2}{*}{ Cerca length } & 158 & 151 & 10 & 17 & 138 & 7 & 4 & 4,32 & $8,26^{a}$ \\
\hline & & \multicolumn{3}{|c|}{ Paratype male } & \multicolumn{3}{|c|}{ Paratype male } & & \\
\hline Structure & Aloparatype & $X$ & SD & $\mathrm{N}$ & $X$ & SD & $\mathrm{N}$ & $\mathrm{F}_{\mathrm{tab}}$ & $\mathrm{F}_{\text {obs }}$ \\
\hline Gonocoxite length & 233 & 231 & 11 & 11 & 307 & 11 & 4 & 4,60 & $166,77^{a}$ \\
\hline Gonocoxite width & 68 & 66 & 4 & 11 & 84 & 8 & 4 & 4,60 & $34,11^{a}$ \\
\hline Gonostyle length & 148 & 145 & 7 & 11 & 185 & 11 & 5 & 4,54 & $82,94^{a}$ \\
\hline Lateral lobe length & 203 & 212 & 15 & 11 & 261 & 21 & 5 & 4,54 & $32,87^{a}$ \\
\hline Lateral lobe width & 28 & 24 & 2 & 11 & 28 & 3 & 5 & 0,05 & $0,0398^{a}$ \\
\hline Ejaculatory pump & 118 & 117 & 7 & 10 & 134 & 4 & 4 & 4,67 & $22,55^{a}$ \\
\hline Genital filament & 540 & 508 & 28 & 10 & 508 & 26 & 4 & 4,60 & 0,04 \\
\hline $\begin{array}{l}\text { Ratio genital filament/ } \\
\text { ejaculatory pump }\end{array}$ & 4,58 & 4,44 & 0,24 & 11 & 3,80 & 0,16 & 4 & 4,67 & $24,59^{a}$ \\
\hline $\begin{array}{l}\text { Internal spine - external } \\
\text { spine inferior }\end{array}$ & 28 & 26 & 4 & 11 & 2 & 5 & 11 & 4,35 & $183,05^{a}$ \\
\hline
\end{tabular}

$a$ : statistically significant; Lateral lobe width: Mann-Whitney Test

\section{REFERENCES}

Andrade Filho JD, Valente MB, Andrade WA, Brazil RP, Falcão AL 2001. Flebotomíneos do Estado de Tocantins, Brasil (Diptera: Psychodidae). Rev Soc Brasil Med Trop 34: 323329.

Galati EAB 1995. Phylogenetic systematics of Phlebotominae (Diptera: Psychodidae) with emphasis on American groups. Bol Dir Malariol Saneam Amb 35 (Supl. 1): 133-142.

Galati EAB 2003. Morfologia, terminologia de adultos e identificação dos táxons da América. In. EF Rangel, R Lainson (eds), Flebotomíneos do Brasil, Fiocruz, Rio de Janeiro, p. 53-175.

Martins AV, Falcão AL, Silva JE 1962. Nota sôbre os Flebotomíneos do Estado de Goiás com a descrição de duas espécies novas e da fêmea de Lutzomyia longipennis (Barretto, 1946) e a redescrição do macho da L. evandroi (Costa Lima e Antunes, 1936) (Diptera, Psychodidae). Rev Bras Malariol Doenças Trop 24: 379-401. 
